\title{
On-the-Fly Model Checking Under Fairness That Exploits Symmetry *
}

\author{
Viktor Gyuris ${ }^{1}$ and A. Prasad Sistla ${ }^{2}$ \\ 1 Mathematics, Statistics and Computer Science Department, The University of \\ Illinois at Chicago, USA \\ 2 Electrical Engineering and Computer Science Department, The University of \\ Illinois at Chicago, USA
}

\begin{abstract}
An on-the-fly algorithm, for model checking under fairness, is presented. The algorithm utilizes symmetry in the program to reduce the state space, and employs new novel techniques that make the onthe-fly modelchecking feasible. The algorithm uses state symmetry and eliminates paralle edges in the reachability graph. Experimental results, demonstrating dramatic reductions in both the running time and memory usage, are presented.
\end{abstract}

\section{Introduction}

The state explosion problem is one of the major bottlenecks in temporal logic model checking. Symmetry based techniques have been proposed in [CFJ93, ES93, ID93] for combating the state explosion problem. In these methods the reachability graph is collapsed by identifying states that are equivalent under symmetry, and model checking is performed on the reduced graph. Although the initial methods of [CFJ93, ES93] could only handle a limited set of liveness properties, a more generalized approach for checking liveness properties under various notions of fairness has been proposed in [ES95]. This method constructs an Annotated Quotient Structure (AQS), computes a product graph and explores the product for existence of fair paths that violate the correctness property.

Many traditional model checking algorithms ([BCG95, G96, HP96, K94]) use on-the-fly techniques to avoid storing the complete reachability graph in the main memory. However, none of these techniques employ symmetry. [ID93] uses onthe-fly techniques together with symmetry for model checking. There the focus is on reasoning about a simple but basic type of correctness, i.e., safety properties expressible in temporal logic CTL by an assertion of the form AG-error.

In this paper, we present an on-the-fly model checking algorithm that checks for correctness under fairness and that exploits symmetry. The algorithm explores the product graph at the same time as it constructs it and terminates early if it finds an incorrect fair computation. Thus, it may terminate early even before it completes the construction of the product graph. Furthermore, it only saves the states in the partially constructed product graph but not the edges.

\footnotetext{
* This work is partly supported by the NSF grants CCR-9623229, CCR-9633536
} 
The paper develops additional theory that leads to new novel techniques that make the on-the-fly model-checking feasible. Our algorithm also uses another technique, called state symmetry [ES93], for reducing the running time as well as memory usage. The on-the-fly model checking algorithm has been implemented and experimental results indicate substantial improvement in performance compared to the original method.

The fairness that we consider in this paper is the traditional weak fairness (a computation is said to be weakly fair if every process is either infinitely often disabled or is executed infinitely often). The algorithm for fairness, given in [ES95], works as follows. It constructs the AQS $\bar{M}$ and then computes the product $\bar{B}$ of $\bar{M}$ and $A$ where $A$ is the automaton that accepts exactly all the incorrect computations. It explores $\bar{B}$ checking for existence of "subtly fair" and "final" strongly connected components; in order to check if a strongly connected component $C$ is subtly fair, it resolves $C$ into a threaded graph $C^{\text {thr }}$ and then checks if each of the connected components in $C^{\text {thr }}$ is "obviously fair", i.e., contains a good node. The nodes of the threaded graph are of the form $(v, j)$ where $v$ is a node in $\bar{B}$ and $j$ is a process index.

Our on-the-fly algorithm works as follows. It incrementally constructs $\bar{B}$ as and when required, and at the same time runs a modified algorithm for computing the strongly connected components $(s c c)$ using depth first search [AHU74]. During the depth first search, with each vertex on the stack, it maintains a partition vector. If $u$ is a state in $\bar{B}$ belonging to the $s c c C$ then the partition vector associated with $u$ captures information about the strongly connected components of the threaded graph $C^{\text {thr }}$ (intuitively, if the $i^{t h}$ and $j^{t h}$ components of the partition vector are equal then it indicates that the nodes $(u, i)$ and $(u, j)$ are in the same $s c c$ in $C^{\text {thr }}$ ). Whenever, an edge to an already visited node in the same $s c c$ is explored then the corresponding partition vectors are merged, and whenever a new vertex in the same $s c c$ is visited then the corresponding partitions are combined. After each combine or merge operation, it is checked if all the components in the partition vector are weakly fair, and if the strongly connected component contains a final automaton state; if so, the algorithm terminates indicating that an incorrect fair computation has been found.

In addition to the above, our algorithm uses state symmetry [ES93] to reduce the size of the constructed AQS. A permutation $\pi \in A u t M$ on process indices is a state symmetry of a representative state $s$, if $\pi(s)=s$. The state symmetry is exploited in different forms in our algorithms. When checking the correctness of the execution of a single process, in [ES93], $\bar{B}$ is constructed as the product $\bar{M} \times I \times A$ where $A$ is the automaton. However, we construct $\bar{B}$ as the product $\overline{M \times I} \times A$; the new product can have potentially fewer states. State symmetry is also used to reduce the number of edges in $\bar{B}$ and $\bar{M}$; especially, parallel edges between the same nodes are eliminated. This reduces memory usage. (For example, in any state $s$ of $\bar{M}$, if many processes are in the same local state then it is enough if we execute one of them and store the corresponding transitions.)

Our paper is organized as follows. Section 2 contains notation and preliminaries. In Sect. 3 we develop the necessary theory and present the on-the-fly 
algorithm. We describe various modifications of the algorithm that take state symmetry into consideration. Section 4 presents experimental results showing the effectiveness of our algorithm and dramatic improvements in time as well as memory usage. Section 5 contains concluding remarks.

\section{Preliminaries}

\subsection{Programs, Processes, Global State Graph}

Let $I$ be a set of process indices and Sym $I$ denote the set of all permutations on $I$. We consider a system $P=\|_{i \in I} K_{i}$ of processes running parallel. Each process $K_{i}$ is a set of transitions. We assume that all variables of $P$ are indexed by indices from $I$ that denote the processes that share the variable. A system $P$, that meets the above description, is called an indexed transition system (or briefly program).

A global state of an indexed transition system is an assignment of values to the variables. We assume that the domain of every variable is finite. This assumption is motivated by the applications and yields that there are only finitely many global states. We can define an indexed graph $M$ on the set of global states that captures the behavior of the program. The indexed global state graph is $M=\left\langle S, R, s_{0}\right\rangle$ where $S$ is the set of global states, $s_{0}$ is the initial state, and $R \subseteq S \times I \times S$ is the transition relation, i.e., $s \stackrel{i}{\rightarrow} t \in R$ if there is a transition in process $i$ that is enabled in state $s$ and its execution leads to state $t$.

\subsection{Annotated Quotient Structure, Threaded Graph}

For a permutation $\pi$, we define the action of $\pi$ on the set of global states. For a global state $s$, we define the global state $\pi(s)$ as follows; for every variable $v_{i_{1}, \ldots, i_{k}}$, its value in the state $s$ is given to the variable $v_{\pi\left(i_{1}\right), \ldots, \pi\left(i_{k}\right)}$ in the state $\pi(s)$; of course, if $v_{\pi\left(i_{1}\right), \ldots, \pi\left(i_{k}\right)}$ is not a variable then $\pi(s)$ is undefined. We say that $\pi$ is an automorphism of the indexed global state graph $M$ if the action of $\pi$ is defined on $S, \pi\left(s_{0}\right)=s_{0}$ and $s \stackrel{i}{\rightarrow} t \in R$ exactly when $\pi(s) \stackrel{\pi(i)}{\rightarrow} \pi(t) \in R$. The set of automorphisms of $M$ is denoted by $A u t M$. Certainly, $A u t M$ is a subgroup of Sym I. Given any subgroup $G$ of $A u t M$, we can define an equivalence relation on $S$. State $s$ is equivalent to $t$ if there is a $\pi \in G$ such that $\pi(s)=t . M$ can be compressed using $G$ as follows. The annotated quotient structure (AQS) for $M$ is $\bar{M}=\left\langle\bar{S}, \bar{R}, s_{0}\right\rangle$ where $\bar{S}$ is a set of representative states that contains exactly one state from each equivalence class of $S / G, \bar{R}=\{s \stackrel{\pi, i}{\longrightarrow} t: \pi \in G, s, t \in$ $\bar{S}, s \stackrel{i}{\rightarrow} \pi(t) \in R\}$.

In order to check correctness of a program under fairness, processes need to be traced in the compressed graph $\bar{M}$, that is, $\bar{M}$ needs to be partially unwound. This leads to the notion of a threaded graph. Let $H=\langle V, E\rangle$ be any graph whose edges are labelled with permutations of a set $I$. The threaded graph $H^{\text {thr }}$ corresponding to $H$ is $\left\langle V \times I, E^{\mathrm{thr}}\right\rangle$ where $E^{\mathrm{thr}}=\{(s, k) \rightarrow(t, l): s \stackrel{\pi}{\rightarrow} t \in$ $E$ for some permutation $\left.\pi, l=\pi^{-1}(k)\right\}$. If $H$ has further labels on its edges then $H^{\text {thr }}$ inherits them. For further details consult [ES95]. 


\subsection{Strongly Connected Subgraphs}

The set of states that appear infinitely often in an infinite computation of a finite state program forms a strongly connected subgraph of $M$. Many properties, such as fairness, can be checked by checking properties of reachable strongly connected components ( $s c c$ for short) of $M$. Let $C$ be a subgraph of $M$. Define $\bar{C}$ to be the image of $C$ in $\bar{M}$, that is, to be $\{s \in \bar{S}: \pi(s) \in C$ for some permutation $\pi\}$.

Lemma 1. 1. If $C$ is a scc of $M$ then $\bar{C}$ is a scc of $\bar{M}$.

2. If $\bar{C}$ is a scc of $\bar{M}$ then there is a scc $D$ of $M$ with $\bar{D}=\bar{C}$.

3. If $\bar{C}$ is a scc of $\bar{M}$ then $\bar{C}^{\text {thr }}$ is a disjoint union of scc of $\bar{M}^{\text {thr }}$.

\subsection{Fairness}

In this paper we consider the traditional weak fairness. A computation is said to be weakly fair if every process is either infinitely often disabled or executed. This condition leads to the following definitions on $M$.

Definition 2. A scc $C$ of $M$ is weakly fair if every process is either disabled in some state of $C$ or executed in $C$. (Process $i$ is executed in $C$ if there are states $s, t$ in $C$ such that $s \stackrel{i}{\rightarrow} t \in R$.)

Definition 3. A scc $C$ of $\bar{M}^{\text {thr }}$ is weakly fair if there is a state $(s, k)$ in $C$ such that process $k$ is disabled in $s$ or $C$ has an edge of type $(s, k) \stackrel{k}{\rightarrow}(t, l)$.

Lemma 4. Let $C$ be a scc of $M$. Then $C$ is weakly fair if and only if $\bar{C}^{\text {thr }}$ consists of weakly fair sces of $\bar{M}^{\mathrm{thr}}$.

\subsection{Model Checking}

Let $A$ be an automaton that accepts exactly the incorrect computations. The problem of checking whether the program $P$ has any incorrect weakly fair computation can be decided by looking at the product graph $B_{0}$ of $M$ and $A$. If $B_{0}$ has a weakly fair $s c c$ with a final automaton state then $P$ has a weakly fair incorrect computation.

Let $i_{1}^{0}, \ldots, i_{k}^{0}$ be the indices that are referred to in $A$. In [ES95], it is shown that we can take the smaller product $\bar{B}_{0}$ of $\bar{M}, I^{k}$ and $A$ and perform the search for a $s c c$ in $\bar{B}_{0}$. Formally, $\bar{B}_{0}=\left\langle\bar{S} \times I^{k} \times A, \bar{R}_{\mathrm{pr}}, s_{\mathrm{pr}}\right\rangle$ where $s_{\mathrm{pr}}=$ $\left(s_{0}, i_{1}^{0}, \ldots, i_{k}^{0}, a_{0}\right), \bar{R}_{\mathrm{pr}}$ consists of edges $\left(s, i_{1}, \ldots, i_{k}, a\right) \stackrel{\pi, l}{\longrightarrow}\left(t, j_{1}, \ldots, j_{k}, a^{\prime}\right)$ such that $s \stackrel{\pi, l}{\longrightarrow} t \in \bar{R}, j_{1}=\pi^{-1}\left(i_{1}\right), \ldots, j_{k}=\pi^{-1}\left(i_{k}\right)$, and the automaton A moves from state $a$ to $a^{\prime}$ on the input gained from $s$ after simultaneously interchanging the indices $i_{1}, \ldots, i_{k}$ with $i_{1}^{0}, \ldots, i_{k}^{0}$ respectively. Lemmas 1 and 4 , mutatis mutandis, hold to $\bar{B}_{0}^{\text {thr }}$.

Theorem 5. $P$ satisfies the complement of the property defined by $A$ if and only if there is no scc $C$ of $\bar{B}_{0}$ such that $C$ contains a final automaton state and $C^{\text {thr }}$ consists of weakly fair sccs. 


\section{On-the-Fly Model Checking}

The original algorithm constructed $\bar{B}_{0}$ together with the threaded graphs $\bar{B}_{0}^{\text {thr }}$. This method can be improved by applying the following three new ideas.

First, note that the symmetry of $M \times I^{k}$ was not fully utilized. Instead of compressing $M$ to $\bar{M}$ and then taking the product with $I^{k} \times A$, we can first compress $M \times I^{k}$ to $\overline{M \times I^{k}}$ and then take the product with $A$. This product graph is denoted by $\bar{B}$. It is possible for two states of the form $\left(s, i_{1}, \ldots, i_{k}\right)$ and $\left(s, j_{1}, \ldots, j_{k}\right)$ to be equivalent and be represented by a single state in $\overline{M \times I^{k}}$. Thus, $\overline{M \times I^{k}}$ is possibly smaller than $\bar{M} \times I^{k}$.

The second improvement is the application of an on-the-fly algorithm. Here we incrementally construct $\bar{B}$ and simultaneously explore it. By this exploration we analyze the threaded graphs without constructing them. If the partially explored $\bar{B}$ contains a required subgraph then the algorithm immediately exits saving further computation time. Because of the on-the-fly nature of the algorithm, we do not need to store the complete $\bar{B}$. Specially, no edges need to be stored.

Finally, the third idea is to use the symmetry of a single global state. Up till this point we did not change the number of edges, $\bar{B}$ has as many edges as $\bar{B}_{0}$. Using state symmetry we can reduce the number of edges by eliminating the redundant parallel ones. Such redundant parallel edges can be removed from $\bar{M}$ also. This results further reduction in memory usage.

For keeping the presentation simple, we assume that we are tracking only one process. Doing so, we do not loose generality. All the results, that are presented below, apply (with the obvious modifications) to the case with many tracked processes. In the actual implementation of the algorithm given below, we used the general case.

\subsection{Compressing $M \times I$}

In Subsect. 2.2 we defined an equivalence relation on $S$. Now, we extend it to $S \times I: u=(s, i)$ and $v=(t, j)$ are equivalent if there is a permutation $\pi \in G$ such that $\pi(u)=v$, that is, $\pi(s)=t$ and $\pi(i)=j$. Let $\vec{S}_{\text {aqsi }}$ be a set of representative states that contains exactly one state from each equivalence class. To ensure that $\bar{S}_{\mathrm{aqsi}}$ and $\bar{S}$ are closely related we adopt the obvious convention

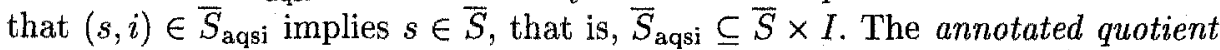
structure with tracked indices (AQSI) of $M \times I$ is $\overline{M \times I}=\left\langle\bar{S}_{\mathrm{aqsi}}, \bar{R}_{\mathrm{aqsi}}, s_{\mathrm{aqsi}}\right\rangle$ where $\bar{R}_{\text {aqsi }}=\left\{(s, i) \stackrel{\pi, l}{\rightarrow}(t, j): s \stackrel{\pi, l}{\rightarrow} t \in \bar{R}, j=\pi^{-1}(i)\right\}$, and $s_{\text {aqsi }}$ is $\left(s_{0}, i_{0}\right)$. In a state $(s, i), i$ is called the tracked process. Note that $\overline{M \times I}$ is the subgraph of $\bar{M} \times I$ spanned by $\bar{S}_{\text {aqsi }}$. Hence $\overline{M \times I}$ is considerably smaller than $\bar{M} \times I$.

Let $\bar{B}$ be the product of $\overline{M \times I}$ and $A$. In the actual algorithm presented below we will construct $\bar{B}$ from $\bar{M}$ (instead of $\overline{M \times I}$ ) and $A$. The reason for that inconvenience is that we do not want to store $\overline{M \times I}$. During the construction of the product graph $\bar{B}$, we can locally construct $\overline{M \times I}$ from the stored $\bar{M}$. This is done in command 7 of the algorithm presented below. 


\subsection{Partitions}

As explained earlier, our on-the-fly model-checking algorithm will explore $\bar{B}$ simultaneously as it constructs it. During this process, in order to analyze the threaded graph without explicitly constructing it, we will maintain a partition of $I$ with each $\bar{B}$ node on the stack; this partition will be represented as an $n$-vector.

First, we would like to adopt the following conventions concerning partitions. We identify equivalence relations and the corresponding partitions on a given set. In that sense, we say that a partition contains another partition if the equivalence relation corresponding to the first partition is a superset of the equivalence relation corresponding to the second partition.

Let $r=(s, l, a)$ be a state in $\bar{B}$ and $C$ be the $s c c$ of $\bar{B}$ that contains $r$. By the analogue version of Lemma $1, C^{\text {thr }}$ is a collection of disjoint sccs. Thus, if a node in $C^{\text {thr }}$ is reachable from both $(r, i)$ and $(r, j)$ then $(r, i)$ and $(r, j)$ are in the same $s c c$ of $C^{\text {thr }}$. Now we define the equivalence relation $\stackrel{*}{\sim}_{r}$ on $I$ as follows:

$$
i \stackrel{*}{\sim}_{r} j \text { if }(r, i) \text { and }(r, j) \text { are in the same component of } C^{\text {thr }} .
$$

It is easy to see that a class of the partition $\stackrel{*}{\sim}_{r}$ identifies a unique component of $C^{\text {thr }}$, and every component of $C^{\text {thr }}$ is identified by a class of ${ }_{\sim_{r}}^{*}$. Thus, we will use these partitions to represent the $\operatorname{sccs}$ of $C^{\text {thr }}$. A class of $\stackrel{*}{\sim}_{r}$ is called weakly fair if the corresponding $s c c$ of $C^{\text {thr }}$ is weakly fair. Note that the tracked process $l$ in $r$ always forms a class of size 1 .

Suppose that if $r$ and $r^{\prime}$ are nodes in the same $s c c$ in $\bar{B}$. The partitions of ${ }^{*}{ }_{r}$ and $\stackrel{*}{\sim}_{r^{\prime}}$ are unfortunately not equal, but one can be obtaind from the other by a permutation in $G$. This problem can be overcome if we use a common referential base. The perfect nominee for this is the initial state $s_{\mathrm{pr}}$ of $\bar{B}$.

Let $T$ be a depth first search spanning tree of $\bar{B}$ with root $s_{\mathrm{pr}}$. For each node $u \in \bar{B}$, let $\pi_{u}$ denote the product of the permutations on the unique $s_{\mathrm{pr}} \sim u$ path in $T$. Now, for each state $r$ in $\bar{B}$, we define an equivalence relation $\sim_{r}$ on $I$ as follows.

$$
i \sim_{r} j \quad \text { if } \quad \pi_{r}^{-1}(i) \stackrel{*}{\sim}{ }_{r} \pi_{r}^{-1}(j) .
$$

With this definition we achieved that $\sim_{r}=\sim_{r^{\prime}}$ whenever $r$ and $r^{\prime}$ are states in the same $s c c$ of $\bar{B}$. Intuitively, $i \sim_{r} j$ indicates that the threads of $\left(s_{\mathrm{pr}}, i\right)$ and $\left(s_{\mathrm{pr}}, j\right)$ enter the same $s c c$ of the threaded graph after they passed $r$.

Next, we show how to compute $\sim_{r}$ by exploring $\bar{B}$ using depth first search. For each edge $e=u \stackrel{\pi, i}{\longrightarrow} v$ in $\bar{B}$, let $\pi_{e}$ denote the permutation $\pi_{u} \circ \pi \circ \pi_{v}^{-1}$. Note that if $e$ is an edge of $T$ then $\pi_{e}$ is the identity permutation. The permutation $\pi_{e}$ satisfies the following property.

Proposition 6. If $e$ is an edge in the scc containing $r$, then $\pi_{e}(i)=j$ implies $i \sim_{r} j$.

Let $\rho$ be a permutation on $I$. We define the orbit partition of $\rho$ on $I$ to be the smallest partition containing $(i, \rho(i))$ for all $i \in I$. Now Claim 6 can be reformulated as: If $e$ is an edge in the scc of $r$, then the orbit partition of $\pi_{e}$ is smaller than or equal to $\sim_{r}$. The following stronger result characterizes $\sim_{r}$. 
Theorem 7. $\sim_{r}$ is the smallest partition that contains the orbit partition of $\pi_{e}$, for every edge $e$ in the strongly connected component of $r$.

The next theorem is a necessary and sufficient condition for checking if a class of $\sim_{r}$ is weakly fair. Let $C$ be the $s c c$ of $r$ in $\bar{B}$.

Theorem 8. A class $K$ of the partition $\sim_{r}$ is weakly fair if and only if there is an $i \in K$ and $u \in C$ such that process $\pi_{u}^{-1}(i)$ is disabled in $u$ or it is executed (that is, there is an edge $u \stackrel{\pi, \pi_{u}^{-1}(i)}{\longrightarrow} v$ in $C$ ).

We have gathered together all the necessary tools to present the on-the-fly algorithm.

\subsection{The Algorithm}

Our algorithm in Table 1 is a modification of the strongly connected component computation using depth first search presented e.g. in [AHU74]. For each vertex $u=(s, l, a)$ of the product graph $\bar{B}$, we maintain the following information.

$u$.dfnum is a unique id (or depth first number) of the node, used for the strongly connected component computation.

$u$. lowlink is the id of a reachable node lower than $u$ itself.

$u$.onstack is a flag indicating that $u$ is still on stack.

$u$.perm is the vector $\pi_{u}$ as defined in the previous subsection.

$u$. partition is an approximation of $\sim u$.

$u$.status is a vector of flags that indicate which partition classes are known to be weakly fair.

$u$. final is a flag that indicates if $u$ is in a $s c c$ that contains a final automaton state. This information is propagated down on the depth first tree.

The variables $u$.dfnum, $u$. lowlink and $u$.onstack are maintained as in the algorithm given in [AHU74]. Command 3 initializes $u$. status and $u$.final appropriately. The two "for" loops in commands 5 and 6 , generate the successors of the $\bar{B}$ state $u$. In command 7 , we construct the product state $v$. To do this, we consider the state $\left(t, \pi^{-1}(l)\right)$ of $\overline{M \times I}$ and find an already generated state of the form $\left(t, l^{\prime}\right)$ and a permutation $\rho$ such that $\left(t, \pi^{-1}(l)\right)$ is equivalent to $\left(t, l^{\prime}\right)$ under $\rho$. If no such equivalent state has already been generated, then $l^{\prime}$ is taken as $\pi^{-1}(l)$ and $\rho$ is taken as the identity permutation. Here the same equivalence checking function can be used as in the construction of $\bar{M}$. (It is to be noted that we need this equivalence checking since we are constructing $\bar{B}$ to be the product of $\overline{M \times I}$ and the automaton $A$, and we are doing this using $\bar{M}$ and $A$; however, if we want to construct $\bar{B}$ to be $\bar{M} \times I \times A$, as in [ES95], then we do not need this equivalence checking, and in this case we may have more states in the resulting $\bar{B}$.)

In command 8 , we check if the edge $u \rightarrow v$ is a non-tree edge (i.e., $v$ has already been visited) and $v$ is in the same $s c c$ as $u$. In this case, it merges the orbit partition of $\pi_{e}$ with $u$.partition. Commands 11 through 14 are executed if the edge $u \rightarrow v$ is a tree edge, i.e., $v$ is constructed (and hence visited) for the 


\section{ON-THE-FLY MODEL CHECKING}

M1. Construct $\bar{M}$.

M2. Construct $A$.

M3. Set $u$ to be the initial state $s_{\mathrm{pr}}$ of $\bar{B}$.

Set $u$.perm to be the identity permutation.

Set the depth-first-counter to zero.

Conduct DF-Search $(u)$.

DF-Search $(u) \quad$ (Note that $u=(s, l, a)$ ).

1. Push $u$ in the stack, set $u$. onstack.

Set $u$.dfnum and $u$.lowlink to the depth-first-counter.

Increase the depth-first-counter.

2. Initialize $u$.partition to be the identity partition.

3. Initialize $u$. status with the information on disabled processes stored in the AQS state $s$.

Set $u$.final if $a$ is a final automaton state.

4. (Idle command. Later modification will use it.)

5. For each automaton transition $a \rightarrow a^{\prime}$ that is enabled in $s$ do

6. For each AQS edge $e=s \stackrel{\pi, i}{\longrightarrow} t$ do

7. Find an already generated AQSI state of the form

$\left(t, l^{\prime}\right)$ that is equivalent to $\left(t, \pi^{-1}(l)\right)$ and

find the permutation $\rho \in G$ that maps $\left(t, \pi^{-1}(l)\right)$ to $\left(t, l^{\prime}\right)$.

Let $v=\left(t, l^{\prime}, a^{\prime}\right)$.

8. $\quad$ If $v$ is already constructed and $v$.onstack is set do

9. Merge $u$.partition with the orbit partition of $\pi_{e}$.

Update $u$.status using that process $i$ was executed.

Update $u$.lowlink to be the minimum of $u$. lowlink and $v$.lowlink.

10. If $v$ is not constructed yet then do

11. Set $v \cdot$ perm $=u$. perm $\circ \pi \circ \rho$.

12. Conduct DF-Search $(v)$.

13. If $v$.onstack is still set then do

14. Combine $v$.partition to $u$.partition.

Combine $v$.status to $u$.status.

Update $u$.status using that process $i$ was executed.

Update $u$.lowlink to be the minimum of $u$.lowlink and $v$.lowlink.

Set $u$.final if $v$.final is set.

15. If all the partition classes are weakly fair (use $u$.status) and $u$.final is set then exit with Yes answer.

16. If $u$.dfnum $=u$. lowlink then do

17. Pop all elements above $u$ (inclusive) from the stack and mark the popped vertices off-stack.

18. Return with No answer. 
first time. In command $11, v$.perm is set; in command $12, \mathrm{DF}$-search is invoked on $v$. If $v$ and $u$ are in the same $s c c$ (indicated by the condition in command 13) then the partitions are combined, the status vector of $u$ is updated and other updates are carried out. After processing the edge $u \rightarrow v$, in command 15, we check if the partially explored $s c c$ containing $u$ is weakly fair and has a final state; if so the algorithm exits with an "yes" answer indicating a fair computation accepted by the automaton $A$ is found. In command 17 , after detecting the $s c c$, we pop all the states of the $s c c$ from the stack.

Theorem 9. The algorithm described above outputs Yes if and only if the original program has a weak fair computation that is accepted by $A$. The algorithm runs in time $O(|\overline{M \times I}| \cdot|I| \cdot|A|)$.

\subsection{State Symmetry, Partition Initialization}

Let $u=(s, l, a)$ be a vertex of the product graph $\bar{B}$. Process $i$ and $j$ are called $u-$ equivalent, denoted by $i \stackrel{*}{\approx}_{u} j$, if there is a permutation $\rho \in G$ such that $\rho(i)=j$ and $\rho(u)=u$. Intuitively, $i \stackrel{*}{\approx} u j$ shows that process $i$ and $j$ are identical in state $u$. Let $u \stackrel{\pi, l}{\longrightarrow} v$ be an edge of $\bar{B}$. Then $\stackrel{\rho \circ \pi, \rho(l)}{\longrightarrow} v$ is also an edge yielding that $\left(v, \pi^{-1}(i)\right)$ is a successor of both nodes $(u, i)$ and $(u, j)$ in the threaded graph $\bar{B}^{\text {thr }}$. Therefore, $(u, i)$ and $(u, j)$ are in the same scc of $\bar{B}^{\text {thr }}$. Hence, $i \stackrel{*}{\sim}_{u} j$.

Lemma 10. The partition $\stackrel{*}{\approx}$ is smaller than $\stackrel{*}{\sim}$.

This fact allows an improvement to the algorithm. First we need to project down $\stackrel{*}{\approx}$ to the common referential base. We define $i \approx_{u} j$ if $\pi_{u}^{-1}(i) \stackrel{*}{\approx}_{u} \pi_{u}^{-1}(j)$. Command 2 in DF-Search can be changed to

$2^{\prime}$. Initialize $u \cdot$ partition to be $\approx_{u}$.

\subsection{Parallel edges in $\bar{B}$}

Let $e=u \stackrel{\pi, l}{\longrightarrow} v$ and $e^{\prime}=u \stackrel{\pi^{\prime}, l^{\prime}}{\longrightarrow} v$ be edges in $\bar{B}$. We call $e$ and $e^{\prime}$ parallel if there is a permutation $\rho \in G$ such that $\rho(u)=u, \rho \circ \pi=\pi^{\prime}, \rho(l)=l^{\prime}$. Surely, being parallel is an equivalence relation on the edges. Let $\bar{R}_{\mathrm{pr}}^{r}$ be a set of representative edges that contains at least one edge from each parallel class. Having the partitions initialized as presented in command $2^{\prime}$, the orbit partition of $\pi_{e^{\prime}}$ does not give any new information after the orbit partition of $\pi_{e}$ was considered. It is reflected in the next lemma.

Lemma 11. If $u$ is in a scc of $\bar{B}$ then $\sim_{u}$ is the smallest partition that contains $\approx_{v}$ (the initial value of $v$.partition) for every $v$ in the scc of $u$ as well as the orbit partition of $\pi_{\mathrm{e}}$ for every edge $e \in \bar{R}_{\mathrm{pr}}^{r}$. 
These ideas can be applied as follows. ¿From each class of $\approx_{u}$ pick a representative process and call it the leader of that class. Put $\bar{R}_{\mathrm{pr}}^{r}=\left\{u \stackrel{\pi, l}{\longrightarrow} v \in \bar{R}_{\mathrm{pr}}\right.$ : $l$ is a leader $\}$. Since every edge is parallel to one that was caused by a leader process, this $\bar{R}_{\mathrm{pr}}^{r}$ is a satisfactory set of representative edges. We introduce the new vector $u$. leader of flags. The next improvement in the algorithm is the introduction of command 4 and the modification of command 6 .

4. Initialize $u$. leader.

5. For each automaton transition $a \rightarrow a^{f}$ that is enabled in $s$ do $6^{t}$. For each AQS edge $e=s \stackrel{\pi, i}{\longrightarrow} v$ if $u$.leader [i] is set do

\subsection{Redundant edges in $\bar{M}$}

In this subsection we show that the edges of $\bar{M}$ can be stored in a more compact way. For that we define state equivalence for every AQS state $s$.

$$
i \stackrel{*}{\approx}_{s} j \text { if there is a } \mu \in G \text { with } \mu(s)=s, \mu(i)=j .
$$

Note that in Subsect. 3.4 we introduced state equivalence for $\bar{B}$ states. The state equivalence relation for $u=(s, l, a)$ was denoted by ${\underset{\sim}{*}}_{u}$. We recall that $i \stackrel{*}{\approx}_{u} j$ if there is a $\mu \in G$ with $\mu(s)=s, \mu(l)=l$ and $\mu(i)=j$. It follows that $i \stackrel{*}{*}_{u} j$ implies $i \stackrel{*}{\approx} j$.

Let $s \stackrel{\pi, i}{\longrightarrow} t$ be an edge of $\bar{M}, \mu(s)=s, \mu(i)=j$. Then $s \stackrel{\mu \circ \pi, j}{\longrightarrow} t$ is an edge as well. This simple observation shows that we need not store both $s \stackrel{\pi, i}{\longrightarrow} t$ and $s \stackrel{\mu \circ \pi, j}{\longrightarrow} t$ provided that $\mu$ can be efficiently computed from $s, i$ and $j$.

We are ready to present the last improvement to our algorithm.

M1'. Construct $\bar{M}$ with the following modifications. When a new node $s$ is created, compute $\stackrel{*}{\approx}$. Define a vector $s$. repr that, for every index $i$, points to a representative of the $\stackrel{*}{\approx}$-class of $i$. By the construction of the edges of $\bar{M}$, store only those edges that are caused by a representative process. (So $s \stackrel{\pi, i}{\longrightarrow} t$ is stored if $s \cdot \operatorname{repr}[i]=i$.)

$6^{\prime \prime}$. For each stored AQS edge $e=s \stackrel{\pi, i}{\rightarrow} t$ and each process $j$ with $s . r e p r[j]=i$ and $u$.leader $[j]$ is set, compute $\mu \in G$ with $\mu(s)=s$ and $\mu(i)=j$, and then do

$11^{i} \quad$ Set $v \cdot$ perm $=u \cdot$ perm $\circ \mu \circ \pi \circ \rho$.

\section{Implementation}

We have developed a prototype of the on-the-fly model checker implementing the above presented algorithm. We used the implemented system to check for the 
correctness of the Resource Controller example with various number of users. We were able to check many properties including the liveness property that every user process that requests a resource will eventually access the resource, and the mutual exclusion property.

We contrasted our new system with the old model checker that implements the results presented in [ES95] on the Resource Controller example. Dramatic improvement was detected in all performance measures as indicated in table 2 below. The product graphs constructed by the old and new model checker are referred to as $\bar{B}_{0}$ and $\bar{B}$ respectively. Each statistics is given as $a / b$ where $a$ and $b$ are the numbers corresponding to the old and new model checker respectively.

\begin{tabular}{|l|c|c|c|}
\hline Liveness Prop. & 10 & 50 & 100 \\
\hline AQS states & $38 / 38$ & $198 / 198$ & $398 / 398$ \\
\hline AQS transitions & $235 / 107$ & $6175 / 1567$ & $24850 / 5642$ \\
\hline AQS const. time (sec) & $0 / 0$ & $16 / 5$ & $149 / 39$ \\
\hline Explored $\bar{B}_{0} / \bar{B}$ states & $385 / 91$ & $9943 / 91$ & $39893 / 91$ \\
\hline $\bar{B}_{0} / \bar{B}$ const. time (sec) & $0 / 0$ & $19 / 0$ & $330 / 0$ \\
\hline Total memory used (kbyte) & $31 / 13$ & $1219 / 216$ & $6878 / 830$ \\
\hline Total CPU time used (sec) & $0 / 0$ & $37 / 6$ & $481 / 42$ \\
\hline \hline Mutual Excl. Prop. & 10 & 50 & 100 \\
\hline AQS states & $38 / 38$ & $198 / 198$ & $398 / 398$ \\
\hline AQS transitions & $235 / 107$ & $6175 / 1567$ & $24850 / 5642$ \\
\hline AQS const. time (sec) & $0 / 0$ & $16 / 5$ & $149 / 39$ \\
\hline Explored $\bar{B}_{0} / \bar{B}$ states & $38 / 38$ & $198 / 198$ & $398 / 398$ \\
\hline $\bar{B}_{0} / \bar{B}$ const. time (sec) & $0 / 0$ & $1 / 0$ & $7 / 2$ \\
\hline Total memory used (kbyte) & $11 / 10$ & $481 / 221$ & $2903 / 857$ \\
\hline Total CPU time used (sec) & $0 / 0$ & $18 / 6$ & $158 / 44$ \\
\hline
\end{tabular}

Table 2. Statistics for the model checker

The liveness property we checked is not satisfied by the Resource Controller. Both model checkers found a fair incorrect computation. ¿From the table, we see that the number of AQS states are the same, while the number of AQS transitions is much smaller in the new model checker due to the use of state symmetry. The number of product states explored in the on-the-fly system is much smaller since it terminated early. On the other hand the original model checker constructed the whole $\bar{B}$ before checking for an incorrect fair computation.

For the mutual exclusion property, both model checkers indicated that the resource controller satisfies the mutual exclusion property. In this case, early termination does not come into effect. Furthermore, since we do not track any process (mutual exclusion is a global property), the number of states explored in $\bar{B}_{0}$ and $\bar{B}$ are the same. However, the number of transitions are much smaller in $\bar{M}$ as well as in $\bar{B}$ due to the effect of state symmetry. The over all CPU time and the memory usage are substantially smaller for the new model checker. 


\section{Conclusions}

In this paper, we have presented an on-the-fly model checking system that exploits symmetry (between states as well as inside a state) and checks for correctness under fairness.

As part of future work, it plan to explore techniques to automatically detect symmetries and integrate these techniques with the model checker. Also, algorithms for checking equivalence of global states under other types of symmetry need to be further explored.

\section{References}

[AHU74] Aho, A. V., Hopcroft, J., Ullman, J. D.: The Design and Analysis of Computer Algorithms. Addison-Wesley (1974)

[BCG95] Bhat, G., Cleaveland, R., Grumberg, o.: Efficient On-the-Fly Modelchecking for CTL. International Conference on Logic in Computer Science, San Diego, California, 1995

[CES83] Clarke, E. M., Emerson, E. A., Sistla, A. P.: Automatic Verification of Finite State Concurrent Programs. using Temporal Logic: A Practical Approach, Proceedings of the ACM Symposium on Principles of Programming Languages, January 1983, Austin, Texas, Also appeared in ACM TOPLAS, April 1986

[CFJ93] Clarke, E. M., Filkorn, T., Jha, S.: Exploiting Symmetry in Temporal Logic Model Checking. 5th International Conference on Computer Aided Verification, Crete, Greece, June 1993.

[ES93] Emerson, E. A., Sistla, A. P.: Symmetry and Model Checking. 5th International Conference on Computer Aided Verification, Crete, Greece, June 1993

[ES95] Emerson, E. A., Sistla, A. P.: Utilizing Symmetry when Model Checking under Fairness Assumptions: An Automata-theoretic Approach. 7th International Conference on Computer Aided Verification, Leige, Belgium, July 1995

[G96] Godefroid, P.: Partial-Order Methods for the Verification of Concurrent Systems. Lecture Notes in Computer Science 1032 Springer, 1996

[HP96] Holzmann, G.J., Peled, D.: The State of SPIN. 8th Intl. Conference on Computer Aided Verification, July 1996

[ID93] Ip, C. N., Dill, D. L.: Better Verification through Symmetry. Intl. Symposium on Computer Hardware Description Languages and their Application, April 1993. Also in Formal Methods in System Design 9 1/2 (1996) 41-75

[K94] Kurshan, R. P.: Computer Aided Verification of Coordinated Processes: The Automata Theoretic Approach. Princeton Univerity Press, Princeton NJ (1994) 